\title{
Plano inclinado no tratamento da mordida cruzada anterior: relato de caso clínico
}

\section{Treatment with inclined plane for anterior crossbite: clinical case report}

Priscilla Bittencourt de Almeida Figueiredo* Rafael Paiva Ferraz ${ }^{* *}$

Vanessa Colares Silva* Jesus Maués Pinheiro Junior ${ }^{* * *}$ Alexandre Roberto Queiroz da Silva ${ }^{* * * *}$ Antonio Irlan Silva****

\section{Resumo}

O diagnóstico e a intervenção ortodôntica precoce feita pela odontopediatra nos casos de má oclusão tanto na dentadura decídua quanto na mista determinam o sucesso do tratamento para cada caso. Objetivo: o presente artigo expõe um caso de mordida cruzada anterior de origem dental na dentadura decídua envolvendo os elementos 51 e 52 em paciente de gênero feminino com quatro anos de idade. Relato de caso: Para o tratamento, realizou-se a confecção de aparelho tipo plano, inclinado em $45^{\circ} \mathrm{em}$ resina acrílica autopolimerizável de forma a permitir uma guia para o descruzamento dessa mordida. A manutenção do aparelho foi feita semanalmente para realizar checagem da oclusão e ajustes necessários. Após duas semanas, o aparelho foi removido e encontrado um resultado satisfatório, com o descruzamento alcançado e o dente em posição de oclusão ideal. Considerações finais: podendo-se, assim, concluir que a utilização do plano inclinado é uma opção eficaz e rápida no tratamento da mordida cruzada anterior na dentadura decídua.

Palavras-chave: Odontopediatria. Má oclusão. Dentição decídua.

\section{Introdução}

Dentre as más oclusões que podem ocorrer na fase de dentadura decídua, a mordida cruzada anterior merece um destaque em virtude da interferência funcional e das alterações que promove no desenvolvimento dentofacial, resultando em deformidades importantes que comprometem a estética e a função do sistema estomatognático ${ }^{1}$.

A mordida cruzada anterior é o posicionamento anormal entre os incisivos, em que um ou mais incisivos superiores posicionam-se lingualmente em relação aos inferiores, quando em relação cêntrica ou habitual ${ }^{2}$. A mordida cruzada anterior pode ser classificada em mordida cruzada anterior dental, funcional ou esquelética, todas com etiologias distintas que servem para diferencia-las ${ }^{3}$. A mordida cruzada anterior dental ocorre devido a uma alteração na inclinação de um ou mais dentes, ou seja, dente superior posicionado por palatina com vestíbulo versão do dente antagonista, estando a relação maxilomandibular correta. Além disso, o paciente apresenta relação molar de classe I. A

Mestre em Odontologia pela Universidade Federal do Pará, especialista em odontopediatria pela ABO Secção Pará, professora das disciplinas de Ortodontia e Unidade Odontológica Infantil do curso de graduação em Odontologia do Centro Superior do Pará (CESUPA), Belém, Pará, Brasil. Acadêmicos do curso de graduação em Odontologia do CESUPA, Belém, Pará, Brasil.

* Doutor em Ortodontia pelo CPO São Leopoldo Mandic, professor das disciplinas de Ortodontia e Unidade Odontológica Infantil do curso de graduação em Odontologia do Centro Superior do Pará (CESUPA), Belém, Pará, Brasil.

*** Cirurgião-dentista graduado pelo curso de graduação em Odontologia do CESUPA, Belém, Pará, Brasil. 
mordida cruzada anterior funcional, ou a pseudo classe III, é aquela em que existe comprometimento das bases ósseas apicais, resultante de uma interferência dental instalada precocemente, que força a mandíbula a mover-se, a fim de obter a máxima intercuspidação, sendo que em relação cêntrica, os incisivos apresentam-se em relação de topo a topo, com os molares separados, porém, em relação molar de classe I. E a mordida cruzada esquelética ocorre devido a uma assimetria ou à falta de harmonia do crescimento ósseo maxilomandibular. Os incisivos superiores estão frequentemente inclinados para frente e os incisivos inferiores estão inclinados para trás, para compensar a displasia esqueletal ${ }^{4-6}$.

A mordida cruzada anterior associada ou não à má oclusão de classe III raramente se autocorrige. Diversos autores ${ }^{3,4,7-10}$ são unânimes em afirmar que sua correção deve ser iniciada assim que o diagnóstico for estabelecido e quando o paciente apresentar maturidade suficiente para entender o problema, podendo colaborar em seu tratamento.

$\mathrm{O}$ tratamento para mordida cruzada anterior depende do número de dentes envolvidos, fase de erupção, característica da oclusão, grau de severidade e colaboração do paciente ${ }^{11}$. Diante desses fatores, temos, como opção de tratamento, os desgastes dentais compensatórios, planos inclinados em acrílico, arco palatino com molas para vestibularização de incisivos, bionator de Balter tipo reverso, mentoneiras, máscaras para tração reversa e os aparelhos ortodônticos com arco progênico modificado ${ }^{10-12}$.

O plano inclinado é um aparelho fixo em resina acrílica autopolimerizável que atua como um plano guia anterior de $45^{\circ}$ em relação ao longo eixo do dente, observando-se a desoclusão dos dentes posteriores. É ativado durante a mastigação e a deglutição do paciente, quando os dentes cruzados tocam o aparelho ${ }^{10,13}$.

Baseado na importância do diagnóstico e na interceptação precoce da mordida cruzada anterior, o presente trabalho irá relatar o caso clínico de paciente infantil diagnosticado com essa patologia, bem como seu tratamento.

\section{Relato de caso}

Paciente do gênero feminino, com quatro anos e quatro meses de idade, apresentou-se na Clínica de odontopediatria do Centro Universitário do Pará (Cesupa) relatando como queixa principal "dentes tortos". Durante a anamnese, não foram reladas alterações sistêmicas ou outro dado significante, e em seguida procedeu-se ao exame clínico, em que se constatou que a paciente apresentava boa saúde oral com ausência de cárie e de doença periodontal. No exame oclusal, observou-se mordida cruzada anterior nos elementos 51 e 52 (Figuras 1 e 2), com relação canino de classe I em ambos os lados e relação terminal dos segundos molares decíduos em plano terminal reto (Figuras 3 e 4). Percebeu-se ainda que a paciente apresentava desgaste com perda de estrutura de esmalte na vestibular do elemento 51. Também foi observada a presença de mordida cruzada no elemento 12 da mãe da paciente, o que pode remeter ao padrão hereditário da mordida cruzada. Em seguida, após a solicitação de radiografias panorâmica e cefalométrica para complementação, chegou-se ao diagnóstico de mordida cruzada anterior do tipo dental dos elementos 51 e 52 .

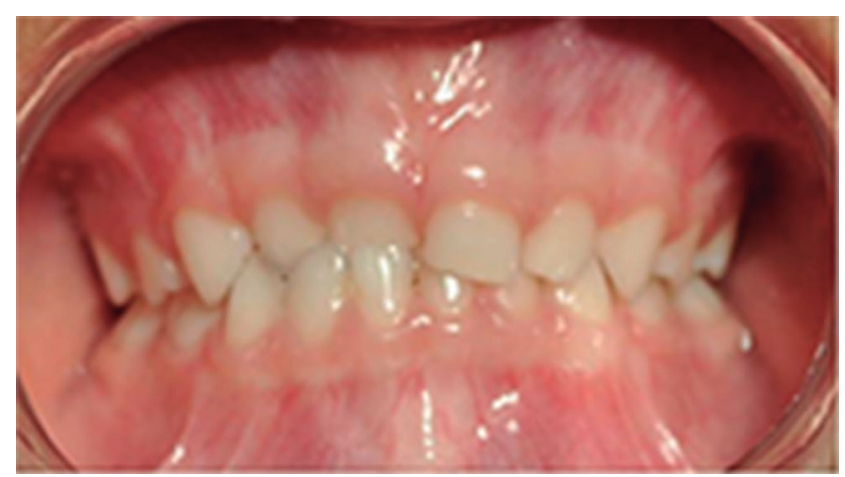

Figura 1 - Mordida cruzada dos elementos 51 e 52 (visão vestibular)

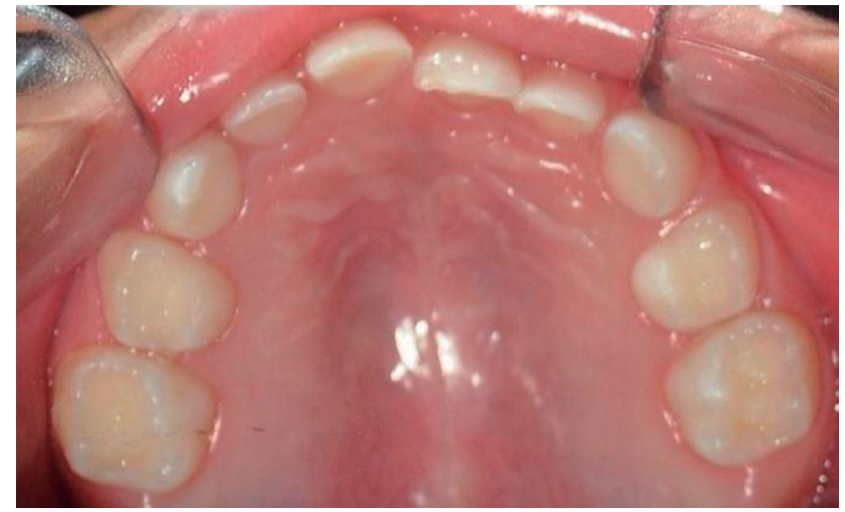

Figura 2 - Visão oclusal com linguoversão dos elementos 51 e 52

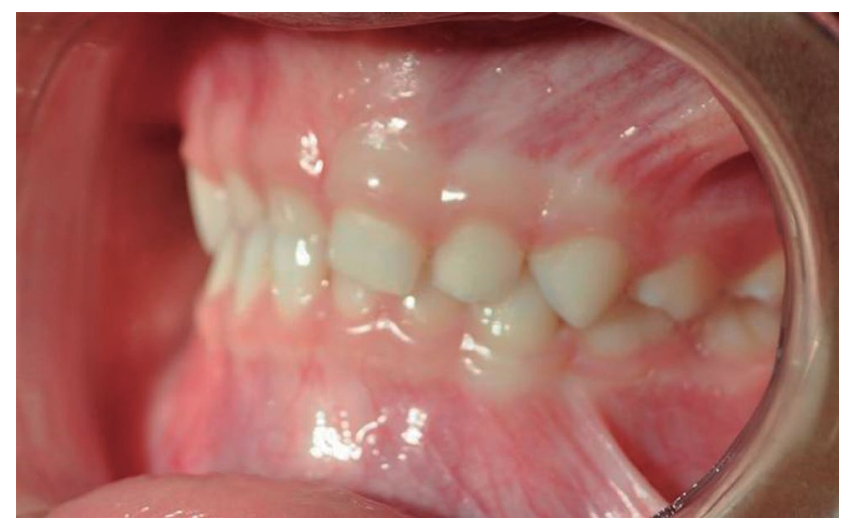

Figura 3 - Relação canino classe I no lado esquerdo 


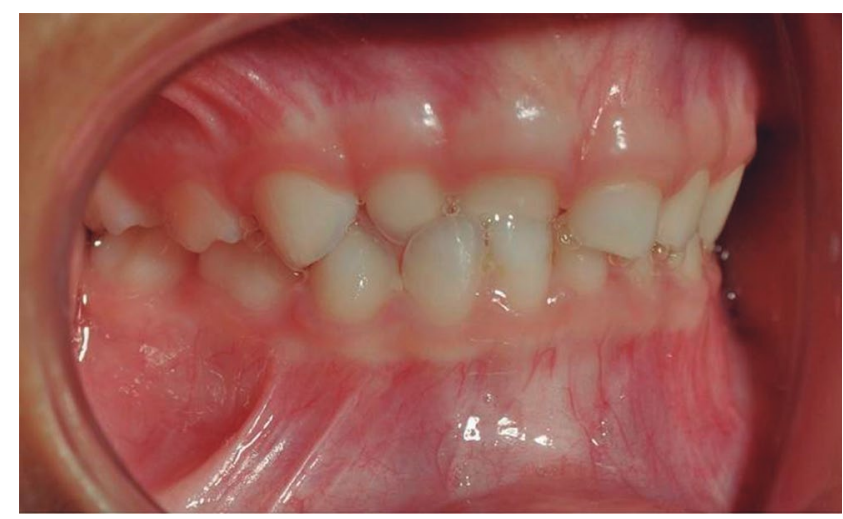

Figura 4 - Relação canino classe I no lado direito

Após uma conversa com os responsáveis e a explanação sobre as alternativas de tratamento, optou-se então pela confecção e pela instalação de um "plano inclinado fixo" devido a pouca idade e à dificuldade de cooperação da paciente, e, também, por ser uma técnica de rápido resultado e com custo reduzido.

Para a confecção do aparelho, inicialmente, foi realizada moldagem de trabalho da paciente e tomada do registro em cera. $\mathrm{O}$ modelo inferior foi isolado, e, em seguida, aplicou-se uma camada de resina acrílica autopolimerizável da marca Vipflash ${ }^{\circledast}$ (Fabricante Vip, Pirassununga, São Paulo, Brasil) sobre os terços médio e incisal, do lado vestibular e palatino de canino a canino inferiores em ângulo de $45^{\circ}$, sem estabelecer contato com o tecido gengival para evitar inflamação. Para cada dente a ser descruzado, foram utilizados dois elementos dentais como apoio no arco inferior. Posteriormente, foi realizado o acabamento e o polimento desse.

Para a instalação do aparelho, utilizou-se a técnica de condicionamento "dizer-mostrar-fazer", obtendo-se, dessa forma, aceitação e colaboração da paciente. Em seguida, foi feito o ajuste do aparelho e logo após a cimentação com ionômero de vidro para cimentação "Meron C" (Figuras 5 e 6), em virtude de suas vantagens como liberação de flúor, boa adesividade, compatibilidade biológica, baixo coeficiente de alteração volumétrica e térmica, baixa solubilidade na boca. Durante a cimentação, foi realizado isolamento relativo e sistema de sucção, indispensáveis para uma cimentação efetiva do aparelho.

Após uma semana de uso, a paciente retornou à clínica, quando se procedeu o exame de controle, observando-se que a mordida ainda não havia descruzado, orientou-se o uso do plano inclinado por mais uma semana. No período de duas semanas, observou-se o descruzamento da mordida e o realinhamento dos elementos 51 e 52. Dessa forma, o aparelho foi removido (Figura 7), e realizou-se a profilaxia para futura restauração do elemento $51 \mathrm{com}$ resina fotopolimerizável z250 na cor A1 (Figura 8).

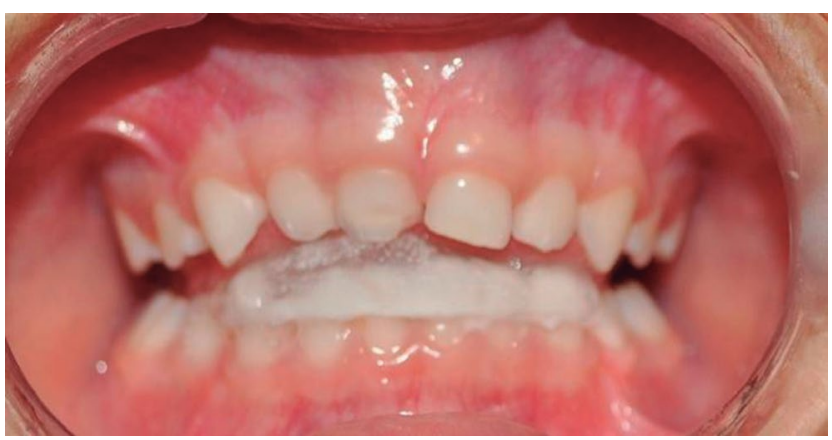

Figura 5 - Aparelho instalado (Visão vestibular)

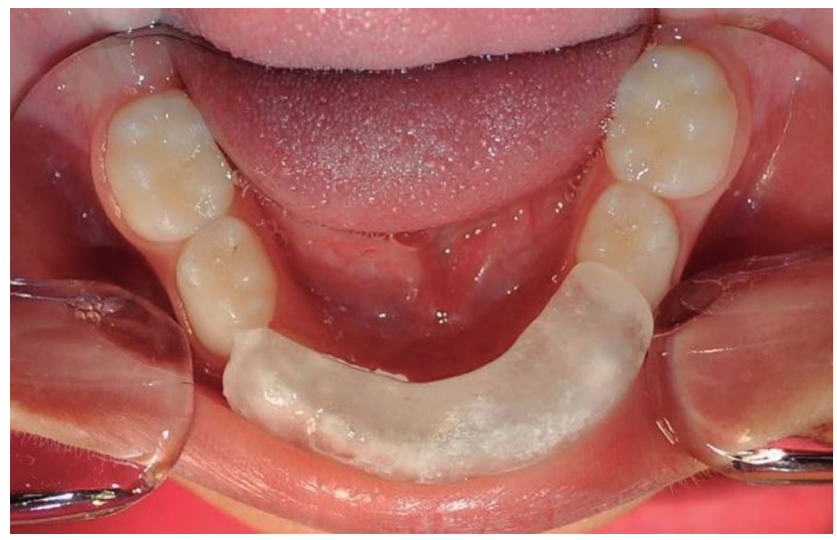

Figura 6 - Aparelho instalado (visão arcada inferior)

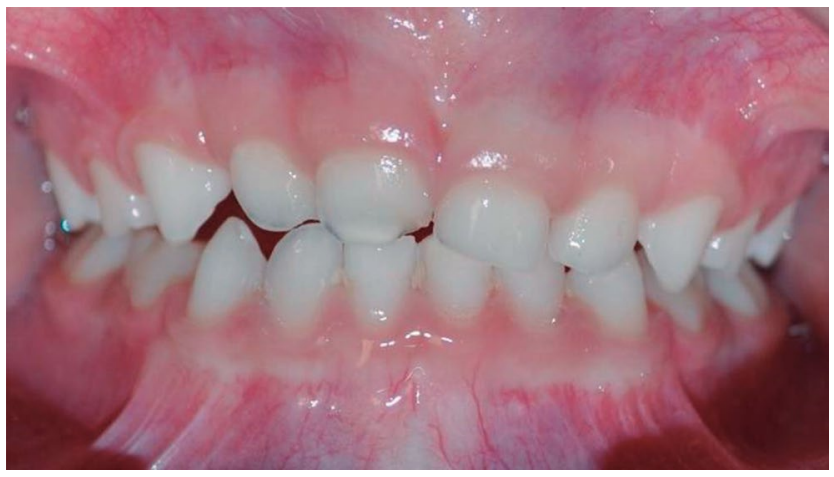

Figura 7 - Aparelho removido e mordida descruzada após 2 semanas

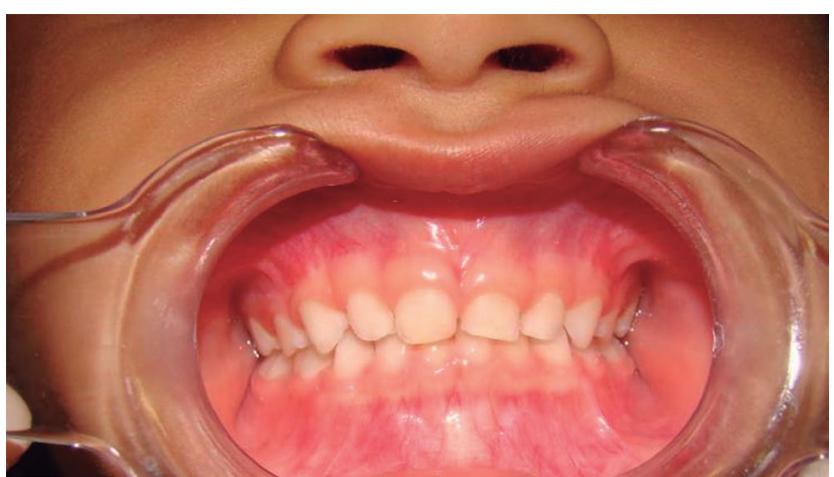

Figura 8 - Após restauração dos elementos 51 


\section{Discussão}

Para diversos autores ${ }^{3,5,11-13}$, a etiologia da mordida cruzada anterior possui vínculos multifatoriais, podendo pertencer à classe de anomalias hereditárias resultantes da discrepância maxilomandibular, geralmente devido a um hiperdesenvolvimento mandibular, a um hipodesenvolvimento maxilar ou em algumas situações à combinação de ambos os fatores. Alguns outros fatores de ordem dental também podem contribuir para a mordida cruzada anterior, como, por exemplo, o trauma na dentadura decídua, a retenção prolongada dos incisivos decíduos, a perda precoce dos incisivos decíduos, os dentes supranumerários, os cistos, os tumores, etc. Os hábitos bucais deletérios como a interposição do lábio superior podem também alterar a inclinação dos incisivos superiores, levando-os para uma posição palatal. A mordida cruzada anterior pode ocorrer ainda por uma interferência oclusal na região anterior, o que caracteriza um deslizamento secundário da mandíbula para anterior ${ }^{10,13}$. No caso da paciente, durante a anamnese não foi relatado nenhum episódio de trauma ou de alterações que pudessem estar relacionados à má oclusão, no entanto, observou-se que a mãe apresentava mordida cruzada do elemento 12 , o que reforça a ideia de padrão hereditário que a má oclusão pode apresentar.

A prevalência de mordida cruzada anterior varia em torno de $7,6 \%$ das crianças, acometendo ainda, cerca de $1 \%$ das crianças entre dois e seis anos de idade. Muitos estudos epidemiológicos têm sido realizados com o objetivo de avaliar a ocorrência da mordida cruzada nas dentições mista e permanente $^{14}$. No que diz respeito à mordida cruzada anterior, estudos no Brasil demonstram que a prevalência varia de $3,3 \%{ }^{15}$ a $18,5 \%{ }^{16}$ e a ocorrência de mordida cruzada posterior varia de $3,0 \%{ }^{17}$ a $13,1 \%{ }^{18}$. Segundo os responsáveis, a paciente apresentava mordida cruzada desde a irrupção dos dentes envolvidos na mordida cruzada, apresentado no momento da consulta inicial quatro anos e quatro meses.

O tratamento da mordida cruzada em idade precoce elimina ou minimiza os danos de um crescimento anormal das bases ósseas e dentoalveolares, evitando problemas periodontais futuros, principalmente no segmento anterior do arco dentário, prevenindo o surgimento de hábitos deletérios como o bruxismo e o desenvolvimento de mordidas cruzadas esqueléticas ${ }^{8,9}$.

Existem diversos dispositivos utilizados na correção da mordida cruzada anterior, fixos ou removíveis. A placa removível com molas vestibularizadoras em resina acrílica é a primeira opção, procedimento considerado o mais biológico e efetivo no movimento ortodôntico do dente. $\mathrm{O}$ paciente é instruído a usar o aparelho 24 horas por dia, removendo-o durante a higienização e a alimentação. A principal desvantagem existente nesse caso é a necessidade de coope- ração do paciente ${ }^{8,13}$. Em virtude da pouca idade e da ausência de colaboração inicial do caso em questão, optou-se pelo uso do plano inclinado, que apesar de ser um recurso pouco utilizado na prática ortodôntica, traz um excelente resultado e mostrou-se um recurso prático, simples e efetivo. No entanto, o uso do plano inclinado apresenta algumas desvantagens como desconforto, dificuldade durante a fala, limitação dietética, estética deficiente, oclusão de dentes sobre o aparelho, os quais se tornam vulneráveis à avulsão ou à luxação e desenvolvem tendência à mordida aberta, se o aparelho for mantido por muito tempo ${ }^{19,20}$. Na confecção do plano inclinado é de suma importância que esse forme um ângulo de $45^{\circ}$ com o longo eixo dos incisivos inferiores, a fim de que não haja resultante vetorial em favor da intrusão do dente cruzado, sendo igualmente importante que haja espaço no arco superior para que ocorra o descruzamento da mordida. Além disso, o recorte do acrílico deve respeitar as margens gengivais para evitar a inflamação do tecido periodontal ${ }^{21-23}$.

\section{Considerações finais}

É de fundamental importância o acompanhamento do odontopediatra durante o desenvolvimento da dentição para que seja realizado um diagnóstico correto e um tratamento precoce nos casos de má oclusão, ajudando consideravelmente no prognóstico. No caso apresentado, as intervenções interceptativas com uso do plano inclinado em resina acrílica solucionou o problema, de maneira eficaz, em pouco tempo e com o mínimo desconforto para a criança, permitindo, dessa forma, que o crescimento e o desenvolvimento ocorram de forma harmoniosa, favorecendo o estabelecimento de uma oclusão satisfatória.

\section{Abstract}

The earlier diagnosis and orthodontic intervention taken by pediatric dentist in cases of malocclusion in deciduous and mixed dentition determine the success of treatment for each case. Objective: This article presents a case of anterior crossbite of dental origin in the primary dentition involving the elements 51 and 52 in a female patient, four years old. Case report: For treatment, there was a cooking appliance type inclined at $45^{\circ}$ in acrylic resin to allow a tab to the unwinding of this bite. The maintenance of the device was made to perform a weekly check of the occlusion and adjustments. After two weeks, the device was removed and found a satisfactory result, with the unwinding achieved tooth position and occlusion ideal. Final considerations: It can be conclude that the use of the inclined plane is an effective and rapid treatment of anterior crossbite in the primary dentition.

Keywords: Pediatric dentistry. Malocclusion. Primary dentition. 


\section{Referências}

1. Litton SF, Ackermann LV, Isaacson RJ, Shapiro B. A genetic study of class III malocclusion. Am J Orthod 1970; 58(6):565-77.

2. Galeão S, Martins LP, Faltin Jr. K, Gandini Jr LG, Pieri LV, Gaspar AMN, et al. Diagnóstico e tratamento precoce da classe III: relato de caso clínico. J Health Sci Inst 2013; 31(1):104-8.

3. Ngan P, Hu AM, Fields Jr HW. Treatment of class III problems begins with differential diagnosis of anterior crossbite. Pediatr Dent 1997; 19(6):386-95.

4. Vianna MS, Casagrande FA, Camargo ES, Oliveira JHG. Mordida cruzada anterior - Relato de um caso clínico. J Bras Ortodon Ortop Facial 2003; 8(44):99-109.

5. Woitchunas DR, Busato C, Tocheto LR, Brockstedt RH. Mordida cruzada anterior: Diagnóstico e tratamento da pseudo classe III: relato de caso clínico. Rev Fac Odontol Univ Passo Fundo 2001; 6(2):23-8.

6. Bayrak S, Tun ES. Treatment of anterior dental crossbite using bonded resin-composite slopers: case reports. Eur J Dent 2008; 2(2):3003-306

7. Mendes AM, Goldner MTA, Capelli Jr. J. Correção da mordida cruzada anterior com arco palatino com mola. Rev Bras Odontol 2009; 66(1):37-40.

8. Park JH, Kim TW. Anterior crossbite correction with a serious of clear removable appliances: a case report. J Esthet Restor Dent 2009; 21:149-59.

9. Tashima AY, Verrastro AP, Ferreira SLM, Wanderley MT, Pinto EG. Tratamento ortodôntico precoce da mordida cruzada anterior e posterior: relato de caso clínico. J Bras Odontopediatr Odontol Bebê 2003; 6(29):24-31.

10. Rossi LB, Pizzol KED, Boeck EM, Lunardi N, Garbin AJI. Correção de mordida cruzada anterior funcional com a terapia de pistas diretas planas: relato de caso. FOL/UNIMEP 2012; 22(2):45-50.

11. Proffit WR. Ortodontia contemporânea. 1.ed. São Paulo: Pancast Editora; 1991.

12. Fernandez MM, Linares AI, Yañezvico RM, Mendonza-Mendonza A, Solano Reina EE. Bone and dentolaveolar anchored dentofacial orthopedias for class III malocclusion: new approaches, similar objectives? Angle Orthodontist 2013; 83(3):540-52

13. Ulsov AV, Bodrumlu EH. Management of anterior dental crossbite with removable appliances. Contemp Clin Dent $2013 ; 4(20): 223-6$

14. Martins DR, Almeida ZR, Dainesi EA. Mordidas cruzadas anterior e posterior. Odontomaster Ortodontia 1994; 1(2):1-19.

15. Marques LS, Barbosa CC, Ramos-Jorge ML, Pordeus IA, Paiva SM. Prevalência da má oclusão e necessidade de tratamento ortodôntico em escolares de 10 a 14 anos de idade em Belo Horizonte, Minas Gerais, Brasil: enfoque psicossocial. Cad Saúde Pública 2005; 21(4):1099-106.

16. Silva Filho OG, Freitas SF, Cavassan AO. Prevalência de oclusão normal e má oclusão na dentadura mista em escolares da cidade de Bauru (São Paulo). Rev Assoc Paul Cir Dent 1989; 43(6):287-90.

17. Almeida MEC, Vedovello Filho M, Vedovello SAS, Lucatto A, Torrezan AT. Prevalência de má oclusão em escolares da rede estadual do município de Manaus, AM, Brasil. RGO 2007; 55(4):389-94

18. Lopes LS, Cangussu MCT. Prevalência e severidade das alterações oclusais em escolares de 12 a 15 anos de Salvador-BA. Rev Ci Med Biol 2005; 4(2):105-12.
19. Sanches LJM, Santos Pinto A, Martins JCR, Raveli DB, Gandini Jr. LG. Tratamento da mordida cruzada anterior com plano inclinado de acrílico. Rev Bras Odontol Rio de Janeiro 1993; 50(2):14-8.

20. Croll TP, Riesenberger RE. Anterior crossbite correction in the primary dentition using fixed inclined planes. Technique and examples. Quintessence Int 1987; 18(12):847-53.

21. Tanaka OM, Maciel JVB, Kreia TB, Ávila ALR, Pithon MM. The anterior dental crossbite: the paradigm of interception in orthodontics. Rev Clin Pesq Odontol 2010; 6(1):71-8.

22. Santos PCF, Monteiro ALB, Lopes BM, Trévia MC, Chaves $\mathrm{AB}$, Brito DAG. Tratamento ortodôntico interceptativo da mordida cruzada anterior: relato de caso clínico. Rev Clin Ortod Dental Press 2012; 11(1):56-68.

23 Maia FA, Ortodontia preventiva e interceptadora. Manual prático. 1.ed. Editora Santos; 2000.

\section{Endereço para correspondência:}

Priscilla Bittencourt Figueiredo

Avenida Serzedelo Correa, 105 apto 703

Bairro Nazaré

66035-400 Belém-PA

Fone: 9188152162

E-mail: priscilla@cesupa.br

Recebido: 11/11/2013. Aceito: 14/05/2014. 